\title{
Quantum Information Processing Using Electron Spins in Quantum Dots
}

\author{
G. BURKARD AND D. LOSS \\ Department of Physics and Astronomy, University of Basel \\ Klingelbergstrasse 82, 4056 Basel, Switzerland
}

\begin{abstract}
We review the theoretical proposal for quantum computing with electron spins in quantum confined structures and discuss the essential requirements for its implementation. The quantum bit is represented by the spin of the electron, as opposed to the charge (orbital) degrees of freedom. In this context, we analyze a number of physical realizations of the elementary building blocks for quantum computation: a universal set of quantum gates, state preparation and measurement. Finally, we discuss the production, transport, and detection of electronic Einstein-Podolski-Rosen pairs, which are an important resource for quantum communication.
\end{abstract}

PACS numbers: 03.67.Lx, 03.67.-a, 72.25.Rb

\section{Introduction}

Computers which operate with quantum states instead of conventional classical information would be capable of efficiently solving some problems for which no efficient classical algorithm is known [1]. In analogy to the smallest unit of information in classical computation, the bit, the memory of a quantum computer can be represented as a collection of quantum two-level systems, named quantum bits, or qubits. At present, there is no working (full-scale) quantum computer, the reason being that a suitable physical realization of qubits is quite hard to find. This is so because the requirements [2] for the implementation of qubits are extremely demanding: Quantum phase coherence needs to be maintained over a long time compared to the length of an elementary step in the computation, in order to allow for quantum error correction. Another requirement is that it should be possible to couple pairs of qubits in a controlled manner in order to carry out elementary quantum logic. In addition to these operations on pairs of qubits, operations on single qubits need to be implemented. It is also required that at the end of a computation, the qubits can be read out by performing a quantum measurement 
separately on each qubit. And finally, the design of the quantum computer should be scalable to a large number of qubits.

Systems involving trapped atoms, cavity QED, or nuclear magnetic resonance (see Ref. [3] for an overview over existing experimental proposals) seem to satisfy all but the scaling requirement from above, and small-scale quantum gate operations with these systems have indeed been demonstrated in experiment. In contrast to the above proposals, it can be speculated that there is a good chance for a solid-state qubit to be scaled up using semiconductor nanotechnology, in a similar manner as for conventional integrated circuits. Several solid-state implementations for quantum computing have been proposed [3]. In this paper, we focus on the idea of using electron spins in coupled semiconductor quantum dots as the qubits of a quantum computer [4], and give an overview of the theoretical work which was carried out in this area (see Fig. 1 for a schematic view of the device under study). Obviously, the spin of the electron is a natural candidate for the quantum bit (qubit) - every spin $1 / 2$ represents precisely one qubit (we identify $|\uparrow\rangle \equiv|0\rangle$ and $|\downarrow\rangle \equiv|1\rangle)$. Treating the spin-orbit interaction and the coupling to the environment as small perturbations this implies that one possible source

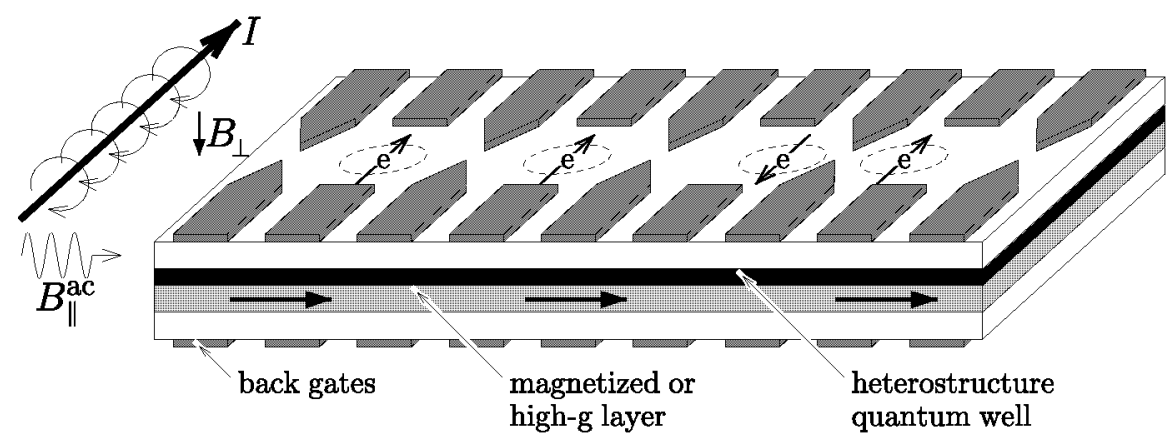

Fig. 1. Array of quantum dots (QDs), indicated by circles, which can be controlled by electrical gating. The electrodes (dark gray) define the confinement potential for the electrons. By changing the gate voltages, electrons can be moved into the magnetized or high- $g$ layer, allowing for spatially varying Zeeman splittings. Local magnetic fields can also be achieved by a current-carrying wire (indicated on the left of the QD array). Now the electron spin in each QD can be addressed individually, e.g. through ESR pulses of an additional in-plane magnetic ac field, because every QD is subject to a distinct Zeeman splitting. This mechanism can be used for single-spin rotations and the initialization step. Changing the exchange coupling $J$ between the QDs can be done by lowering the tunnel barrier between the dots (the two rightmost QDs are drawn schematically as tunnel-coupled). 
of errors in quantum computation, namely the leakage into logically "undefined" parts of the Hilbert space, has already been avoided.

The motivation for doing quantum computing comes from its potential to outperform classical computers [1]. This is possible through quantum algorithms which make use of the quantum memory's ability to exist in quantum superpositions and to undergo a unitary time evolution $U\left|\Psi_{\text {in }}\right\rangle=\left|\Psi_{\text {out }}\right\rangle$, where $\left|\Psi_{\text {in }}\right\rangle$ denotes the "input" state which is prepared before the computation starts and $\left|\Psi_{\text {out }}\right\rangle$ is the final state, which is subsequently measured in order to obtain the result of the computation. A very useful fact is that all possible quantum algorithms can be implemented by concatenating one- and two-qubit gates [5], therefore it is sufficient when we discuss these two types of operations below.

In addition to satisfying all requirements necessary for a scalable quantum computer, a spin-qubit, being attached to a mobile electron, can be transported along conducting wires [6]. An interesting application of this possibility appears to be the creation (say, in coupled quantum dots or near a superconductor-normal interface [7]) and transport of spin-entangled electrons which act as Einstein-Podolsky-Rosen (EPR) pairs. Such EPR pairs represent one of the fundamental resources for quantum communication [1]. We include a discussion of the use of electronic EPR pairs for quantum communication in this article and show that such EPR pairs can be transported and detected in transport and noise measurements in electronic nanostructures [8]. Entangled pairs of particles have already been produced and used for various tasks in quantum communication in several experiments in quantum optics (see e.g. Ref. [9]). For many applications, a disadvantage of the sources which are presently used there (parametric downconversion) is that entangled particles can only be produced in a stochastic manner and at a very low rate. Thus there is a great demand for deterministic and fast sources of EPR pairs. One candidate system for a source of entangled (localized or delocalized) spin qubits would be coupled quantum dots $[6,4]$. A second possibility is the use of $s$-wave superconductor-normal junctions as a source of spin-entangled electrons, exploiting the fact that the Cooper pairs are in a singlet (and therefore entangled) state $[6,7]$.

\section{Coherence}

At first sight, arbitrary quantum two-level systems could serve as qubits. However, in all practical cases, quantum systems are never completely isolated from their environment. The unavoidable coupling to the environment is the origin of decoherence which creates errors in a quantum computation. If these errors are rare enough, they can be corrected using quantum error correction [1]. Therefore, only systems with slow decoherence are interesting for quantum computation. It is important to distinguish the relaxation time $T_{1}$ and the (transverse) decoherence time $T_{2}$. The latter is usually shorter, and therefore more relevant 
for quantum computing. If the spin-orbit coupling is weak then the spin coherence time - the time over which the phase of a superposition of spin-up and spin-down states is well defined - can be completely different from the charge coherence time (as determined e.g. from measurements of the weak localization or the Aharonov-Bohm effect), and in fact it is known from magneto-optical experiments in bulk $n$-doped GaAs and in CdSe quantum dots that they can be orders of magnitude longer $[10,11]$. These findings motivate the use of the spin as the qubit in these structures rather than charge [4].

We have proposed to measure $T_{2}$ of a single spin in such a device directly via a transport experiment by applying electron spin resonance (ESR) techniques [12]. The idea is to extract the decoherence time $T_{2}$ by probing the sequential tunneling current through the quantum dot which is attached to two leads in the presence of an applied ESR field. We assume a situation in which the Zeeman splitting in the leads is negligible, but on the dot we have $g \mu_{\mathrm{B}} B>\Delta \mu$ and $g \mu_{\mathrm{B}} B>k_{\mathrm{B}} T$. This can be achieved e.g. by using materials with different g-factors for the dot and the leads. Then, the stationary master equation for the reduced density matrix of the quantum dot in the basis $|\uparrow\rangle,|\downarrow\rangle,|S\rangle=(|\uparrow \downarrow\rangle-|\downarrow \uparrow\rangle) / \sqrt{2}$ (with corresponding energies $0=E_{\uparrow}<E_{\downarrow}<E_{S}$ ) is derived. We can assume that the triplet is higher in energy and does not contribute to the sequential tunneling current. In the regime $E_{S}>\mu_{1}>E_{S}-g \mu_{\mathrm{B}} B>\mu_{2}$ the current is blocked in the absence of the ESR field due to spin conservation. Only in the presence of the ESR field there can be a sequential tunneling current; after some calculation we find for the stationary current $[12]$

$$
I(\omega) \propto \frac{V_{\downarrow \uparrow}}{\left(\omega-g \mu_{\mathrm{B}} B\right)^{2}+V_{\downarrow \uparrow}^{2}}
$$

where the width of the resonance at $\omega=g \mu_{\mathrm{B}} B$ is given by the total spin decoherence rate $V_{\downarrow}=\left(W_{S \uparrow}+W_{S \downarrow}\right) / 2+1 / T_{2}$. Here, $W_{S \sigma}$ denotes the rate for the transition from the state $|\sigma\rangle=|\uparrow\rangle,|\downarrow\rangle$ to the singlet $|S\rangle$ due to the tunnel coupling to the leads. Therefore, the inverse of the observed line width $1 / V_{\downarrow} \uparrow$ represents a lower bound for the intrinsic single-spin decoherence time $T_{2}$. For finite temperatures, in the linear response regime $\Delta \mu<k T$ the current has roughly the standard sequential tunneling peak shape $\cosh ^{-2}\left[\left(E_{S}-E_{\downarrow}-\mu\right) / 2 k_{\mathrm{B}} T\right]$ where $\mu=\left(\mu_{1}+\mu_{2}\right) / 2$, while the width of the resonance given in Eq. (1) remains unaffected.

Although theory can only give us some ideas about the mechanisms for decoherence rather than reliable quantitative predictions of the decoherence times, we report here on recent calculations identifying the dominant spin-orbit effects in GaAs quantum dots leading to unusually low phonon-assisted spin-flip rates, which suggest long spin-decoherence times. In Ref. [13] the spin-flip processes which accompany phonon-assisted transitions between different discrete energy levels (or Zeeman sublevels) in GaAs electron quantum dots were studied within 
a one-electron Hamiltonian derived from the Kane model. Several different mechanisms originating from spin-orbit coupling are shown to be responsible for such processes and the rates $\Gamma=T_{1}^{-1}$ for all mechanisms are evaluated in the presence or absence of a magnetic field. It turns out that the most effective spin-flip mechanisms in the $2 \mathrm{D}$ case are related to the broken inversion symmetry, either in the elementary crystal cell or at the heterointerface. The spin relaxation of $2 \mathrm{D}$ electrons localized in quantum dots is much smaller than that of delocalized electrons because the contribution of lowest order in the spin-orbit coupling constant $\beta$ vanishes due to the boundedness of the wave function. Therefore, the contributions to the spin-flip rate proportional to $\beta^{2}$ are either absent or suppressed by additional small parameters (e.g. related to the size quantization in the $z$ direction). A finite Zeeman splitting also leads to contributions $\propto \beta^{2}$,

$$
\Gamma \simeq \Gamma_{0}(B) \frac{m \beta^{2}}{\hbar \omega_{0}}\left(\frac{g \mu_{\mathrm{B}} B}{\hbar \omega_{0}}\right)^{2},
$$

where $\hbar \omega_{0}$ is the distance between the orbital levels in the dot, $\Gamma_{0}(B)$ is the inelastic rate without a spin flip. Spin-flip transitions between the Zeeman sublevels (with energy spacing smaller than $\hbar \omega_{0}$ ) occur at a rate which is at low temperature proportional to the fifth power of the Zeeman splitting,

$$
\Gamma \simeq \frac{\left(g \mu_{\mathrm{B}} B\right)^{5}}{\hbar\left(\hbar \omega_{0}\right)^{4}} \Lambda_{\mathrm{p}},
$$

where $\Lambda_{\mathrm{p}} \propto \beta^{2}$ characterizes the strength of the effective spin-piezo-phonon coupling in the heterostructure and ranges from $\approx 7 \times 10^{-3}$ to $\approx 6 \times 10^{-2}$ depending on $\beta$ (e.g. $\Gamma \approx 1.5 \times 10^{3} \mathrm{~s}^{-1}$ for $\hbar \omega_{0}=10 \mathrm{~K}$ and $B=1 \mathrm{~T}$ ). It is also shown that two-phonon contributions to the spin-flip transitions between the Zeeman sublevels are irrelevant at temperatures $k_{\mathrm{B}} T \ll \hbar \omega_{0}$.

\section{Initialization}

Before a typical quantum computation is started, initialized qubits are required, i.e. qubits in a well defined state such as spin up, $|\uparrow\rangle \equiv|0\rangle$. This can be done by exposing all spins in the quantum register to a large magnetic field $g \mu_{\mathrm{B}} B \gg k T$ and allowing them to relax to the ground state. This completely polarized state corresponds to the logical state $|0000 \ldots\rangle$ in which all qubits are set to zero. The required magnetic field can be applied locally or realized by forcing the electrons (via external gates) into a magnetized layer/dot, into a layer with a different effective g-factor [4,6] or with polarized nuclear spins (Overhauser effect) [14] etc., see also Fig. 1. Polarized electrons can also be injected into an empty quantum dot if a spin-polarized current can be produced, such as by spin-polarizing materials $[15,16]$ or by spin-filtering with the help of another dot [17]. Starting from the completely polarized state $|0000 \ldots\rangle$, one can create arbitrary (product) input states by applying the single-spin rotations described in Sec. 6 . 


\section{Laterally coupled quantum dots}

In coupled quantum dots, there is a mechanism which allows for the controlled interaction of two spin-qubits, resulting from the combined action of the Coulomb interaction and the Pauli exclusion principle. The ground state of two coupled electrons in the absence of a magnetic field is a spin singlet, while the first excited state in the presence of sufficiently strong Coulomb repulsion is a spin triplet. Further excited states are separated from these two lowest states by an energy gap, given either by the Coulomb repulsion or the single-particle confinement. Thus, the low-energy dynamics of the two excess electrons in tunnel-coupled quantum dots can be described by the effective Heisenberg spin Hamiltonian

$$
H_{\mathrm{s}}(t)=J(t) \boldsymbol{S}_{1} \cdot \boldsymbol{S}_{2} .
$$

Here, $J(t)$ denotes the exchange coupling between the two spins $\boldsymbol{S}_{1}$ and $\boldsymbol{S}_{2}$ which is given by the separation in energy between the triplet and the singlet. Assuming that $J$ is zero ("switched off") except for a short duration $\tau_{\mathrm{s}}$ ("pulse") with the restriction $\int_{0}^{\tau_{\mathrm{s}}} \mathrm{d} t J(t) / \hbar=J_{0} \tau_{\mathrm{s}} / \hbar=\pi(\bmod 2 \pi)$, we obtain a time evolution $U(t)=T \exp \left(\mathrm{i} \int_{0}^{t} H_{\mathrm{s}}(\tau) \mathrm{d} \tau / \hbar\right)$ which corresponds to the "swap" gate $U_{\mathrm{sw}}$, interchanging the states of qubit 1 and 2 . Swapping qubits is not sufficient for quantum computation; however, the so-called square-root-of-swap $U_{\mathrm{sw}}^{1 / 2}$, obtained under the condition

$$
\int_{0}^{\tau_{\mathrm{s}}} \mathrm{d} t \frac{J(t)}{\hbar}=\frac{J_{0} \tau_{\mathrm{s}}}{\hbar}=\frac{\pi}{2}(\bmod 2 \pi)
$$

turns out to be a universal quantum gate when combined with single-qubits rotations (see Sec. 6), i.e. arbitrary quantum evolutions (algorithms) can be constructed from these elements. The universality of $U_{\mathrm{sw}}^{1 / 2}$ is proven by constructing the known universal gate XOR [18], through combination of $U_{\mathrm{sW}}^{1 / 2}$ and single-qubit operations $\exp \left(\mathrm{i} \pi S_{i}^{z} / 2\right)$, applied in the sequence [4] $U_{\mathrm{XOR}}=\mathrm{e}^{\mathrm{i}(\pi / 2) S_{1}^{z}} \mathrm{e}^{-\mathrm{i}(\pi / 2) S_{2}^{z}}$ $\times U_{\mathrm{sw}}^{1 / 2} \mathrm{e}^{\mathrm{i} \pi S_{1}^{z}} U_{\mathrm{sw}}^{1 / 2}$. Thus, the study of general quantum computation can be reduced to the study of single-spin rotations and the exchange mechanism, i.e. how $J(t)$ can be controlled experimentally. The following investigation of the exchange mechanism is based on the idea to change $J(t)$ can be "switched off" and "switched on" by raising or lowering the tunneling barrier between the dots. In addition to the barrier, there are other external parameters which can be used to control the exchange coupling $J(t)$.

We consider first a system of two coupled quantum dots in a two-dimensional electron gas (2DEG), containing one (excess) electron each. The arrangement of the dots is as depicted in Fig. 1, and we require that the distance $2 a$ between the dots is sufficiently small, allowing the electrons to tunnel between the dots (for a lowered barrier). The model Hamiltonian for this system is

$$
H=\sum_{i=1,2} h_{i}+C+H_{\mathrm{Z}}=H_{\mathrm{orb}}+H_{\mathrm{Z}},
$$


where the single-electron dynamics in the 2DEG ( $x y$-plane) is described through

$$
h_{i}=\frac{1}{2 m}\left[\boldsymbol{p}_{i}-\frac{e}{c} \boldsymbol{A}\left(\boldsymbol{r}_{i}\right)\right]^{2}+V\left(\boldsymbol{r}_{i}\right),
$$

with $m$ being the effective mass and $V\left(r_{i}\right)$ the confinement potential as given below. A magnetic field $\boldsymbol{B}=(0,0, B)$ is applied along the $z$-axis, which couples to the electron spin through the Zeeman interaction $H_{\mathrm{Z}}$ and to the charge through the vector potential $\boldsymbol{A}(\boldsymbol{r})=\frac{B}{2}(-y, x, 0)$. The screening length $\lambda$ in almost depleted regions (like few-electron quantum dots) can be expected to be much larger than the screening length in bulk 2DEG regions (where it is $40 \mathrm{~nm}$ for GaAs). For small quantum dots, say $\lambda \gg 2 a \approx 40 \mathrm{~nm}$, we therefore have to consider the unscreened Coulomb interaction $C=e^{2} / \kappa\left|\boldsymbol{r}_{1}-\boldsymbol{r}_{2}\right|$, where $\kappa$ is the static dielectric constant. The confinement and tunnel-coupling in Eq. (7) for laterally aligned dots is modeled by the quartic potential

$$
V(x, y)=\frac{m \omega_{0}^{2}}{2}\left[\frac{1}{4 a^{2}}\left(x^{2}-a^{2}\right)^{2}+y^{2}\right]
$$

with the inter-dot distance $2 a$ and $a_{\mathrm{B}}=\sqrt{\hbar / m \omega_{0}}$ the effective Bohr radius of the dot. Thus, at large separation $a \gg a_{\mathrm{B}}$ the dots are modeled as two harmonic wells with frequency $\omega_{0}$. This is justified by the experimental evidence that the low-energy spectrum of single dots is well described by a parabolic confinement potential [19].

We consider only the two lowest orbital eigenstates of $H_{\text {orb }}$, leaving us with one symmetric (spin-singlet) and one antisymmetric (spin-triplet) orbital state. The spin state for the singlet is $|S\rangle=(|\uparrow \downarrow\rangle-|\downarrow \uparrow\rangle) / \sqrt{2}$, while the triplet spin states are $\left|T_{0}\right\rangle=(|\uparrow \downarrow\rangle+|\downarrow \uparrow\rangle) / \sqrt{2},\left|T_{+}\right\rangle=|\uparrow \uparrow\rangle$, and $\left|T_{-}\right\rangle=|\downarrow \downarrow\rangle$. At sufficiently low temperatures $k T \ll \hbar \omega_{0}$, higher lying states are frozen out and $H_{\text {orb }}$ can be replaced by the spin Hamiltonian, Eq. (4), and the exchange energy $J=\epsilon_{\mathrm{t}}-\epsilon_{\mathrm{s}}$ is given as the difference between the triplet and singlet energy. For the calculation of these energies, the analogy between atoms and quantum dots can be used. Using the Heitler-London ansatz from molecular physics (with ground-state single-dot orbitals) we obtain [14]:

$$
\begin{aligned}
J= & \frac{\hbar \omega_{0}}{\sinh \left(2 d^{2} \frac{2 b-1}{b}\right)}\left\{\frac{3}{4 b}\left(1+b d^{2}\right)\right. \\
& \left.+c \sqrt{b}\left[\mathrm{e}^{-b d^{2}} I_{0}\left(b d^{2}\right)-\mathrm{e}^{d^{2}(b-1 / b)} I_{0}\left(d^{2}(b-1 / b)\right)\right]\right\},
\end{aligned}
$$

where $d=a / a_{\mathrm{B}}$ is the dimensionless distance between the dots, $b=B / B_{0}=$ $\sqrt{1+\omega_{\mathrm{L}}^{2} / \omega_{0}^{2}}$ the magnetic compression factor, $\omega_{\mathrm{L}}=e B / 2 m c$ the Larmor frequency, and $I_{0}$ denotes the zeroth-order Bessel function. The first term in Eq. (9) originates from the confinement potential, while the terms proportional to the parameter $c=\sqrt{\pi / 2}\left(e^{2} / \kappa a_{\mathrm{B}}\right) / \hbar \omega_{0}$ come from the Coulomb interaction $C$. The term with the leading minus sign is the exchange term. We are mainly interested in the 

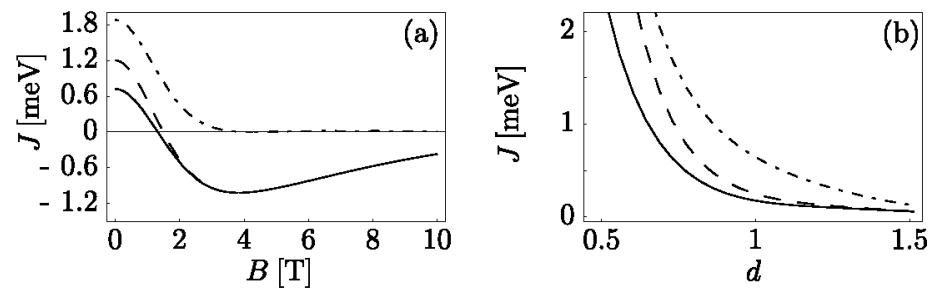

Fig. 2. Exchange coupling $J$ (full line) for quantum dots in a GaAs structure with confinement energy $\hbar \omega=3 \mathrm{meV}$ and $c=2.42$. We plot the conventional short-range Hubbard result $J=4 t^{2} / U$ (dashed-dotted line) and the extended Hubbard result [14] $J=4 t^{2} / U+V$ (dashed line) for comparison. In (a), we plot $J$ as a function of the magnetic field $B$ at fixed inter-dot distance $d=a / a_{\mathrm{B}}=0.7$, while in (b) we vary the inter-dot distance $d=a / a_{\mathrm{B}}$ at zero field $(B=0)$.

weak coupling limit $\left|J / \hbar \omega_{0}\right| \ll 1$, where the ground-state Heitler-London ansatz is self-consistent. We refer to Fig. 2 for a plot of $J$ as given in Eq. (9) as a function of $B$ and $d$. Note that $J(B=0)>0$, i.e. the singlet is the ground state, which is generally true for a two-particle system with time-reversal invariance. Over a wide range of the parameters $c$ and $a$, it can be observed that the sign of $J(B)$ changes from positive to negative at a finite value of $B$ (for the parameters chosen in Fig. $2 \mathrm{a}$ at $B \approx 1.3 \mathrm{~T}$ ). Furthermore, $J$ is suppressed exponentially either by compression of the electron orbitals through large magnetic fields $(b \gg 1)$, or by large distances between the dots $(d \gg 1)$, where in both cases the orbital overlap of the two dots is reduced. This exponential suppression, contained in the $1 /$ sinh prefactor in Eq. (9), is partly compensated by the exponentially growing exchange term $\propto \exp \left[2 d^{2}(b-1 / b)\right]$. Altogether, $J$ decays exponentially as $\exp \left(-2 d^{2} b\right)$ for large $b$ or $d$. Since the sign reversal of $J$ at the point of a singlet-triplet crossing results from the long-range Coulomb interaction, it is not contained in the standard Hubbard model which takes only short-range interaction into account. The latter predicts $J=4 t^{2} / U>0$ in the limit $t / U \ll 1$ (see Fig. 2). Further refinement of the Heitler-London result Eq. (9) was achieved by taking higher levels and double occupancy of the dots into account (implemented in a Hund-Mullikan approach), which leads to qualitatively similar results [14], in particular concerning the singlet-triplet crossing. Numerical calculations which take more single-particle levels into account [20] have confirmed the results presented here.

\section{Vertically coupled quantum dots}

Vertically tunnel-coupled quantum dots have been produced in multilayer self-assembled quantum dots (SAD) [21] as well as in etched mesa heterostructures [22]. In order to investigate quantum gate operations in these structures we apply the same methods as described in the previous section for laterally coupled 


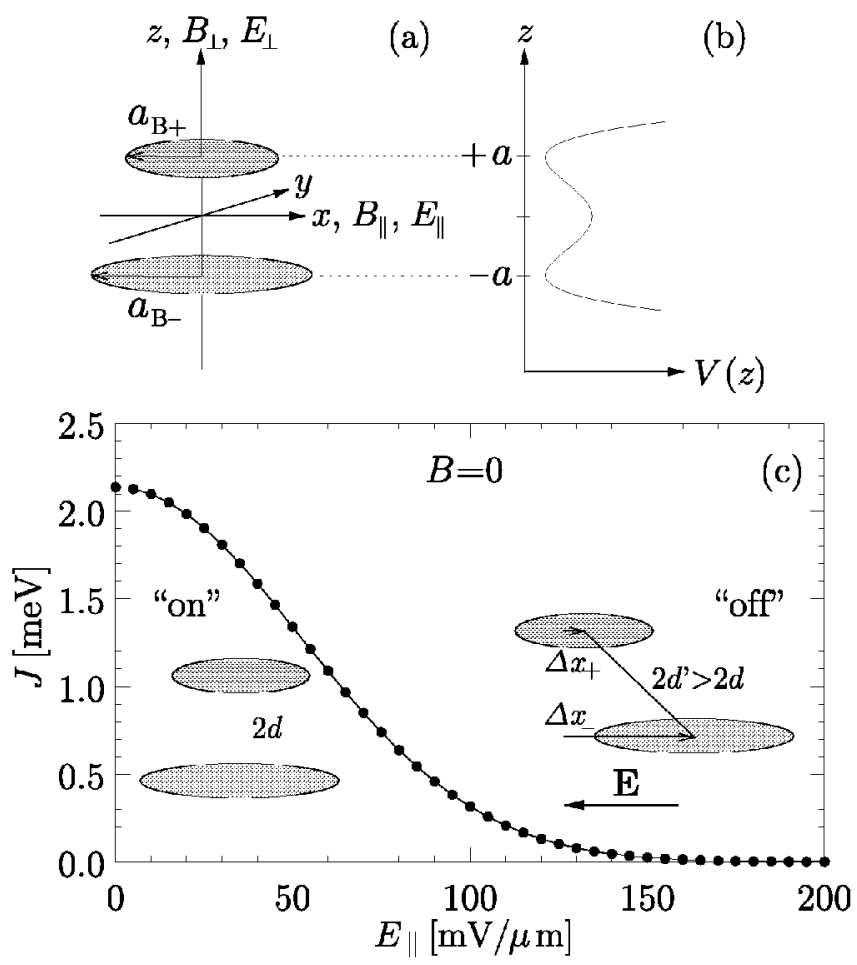

Fig. 3. (a) Schematic drawing of two vertically coupled quantum dots having different lateral radii $a_{\mathrm{B}+}$ and $a_{\mathrm{B}-}$. Magnetic and electric fields can be applied either in-plane $\left(B_{\|}, E_{\|}\right)$or perpendicularly $\left(B_{\perp}, E_{\perp}\right)$. (b) Quartic double-well potential used for modeling the vertical confinement $V_{\mathrm{v}}$. (c) Mechanism for switching of the spin-spin coupling between dots of different size by means of an in-plane electric field $E_{\|}$at $B=0$. The parameters for this plot are $\hbar \omega_{z}=7 \mathrm{meV}, d=1, \alpha_{0+}=a_{\mathrm{B}}^{2} / a_{\mathrm{B}+}^{2}=1 / 2$ and $\alpha_{0-}=a_{\mathrm{B}}^{2} / a_{\mathrm{B}-}^{2}=1 / 4$. Therefore, $E_{0}=\hbar \omega_{z} / e a_{\mathrm{B}}=0.56 \mathrm{mV} / \mathrm{nm}$ and $A=$ $\left(\alpha_{0+}^{2}-\alpha_{0-}^{2}\right) / 2 \alpha_{0+}^{2} \alpha_{0-}^{2}=6$. The exchange coupling $J$ decreases exponentially on the scale $E_{0} / 2 A=47 \mathrm{mV} / \mu \mathrm{m}$ for the electric field. The exchange coupling is switched "on" for $E_{\|}=0$ and "off" for $E_{\|} \approx 150 \mathrm{mV} / \mu \mathrm{m}$.

dots, but now we extend the Hamiltonian Eq. (7) from two to three dimensions and use a three-dimensional confinement potential $V=V_{l}+V_{\mathrm{v}}[23]$. We implement the vertical confinement $V_{\mathrm{v}}$ as a quartic potential similar to Eq. (8), with curvature $\omega_{z}$ at $z= \pm a$ (see Fig. $3 \mathrm{~b}$ ), implying an effective Bohr radius $a_{\mathrm{B}}=\sqrt{\hbar / m \omega_{z}}$ and a dimensionless distance $d=a / a_{\mathrm{B}}$. We have modeled a harmonic potential for the lateral confinement, while we have allowed for different sizes of the two dots $a_{\mathrm{B} \pm}=\sqrt{\hbar / m \alpha_{0 \pm} \omega_{z}}$. This results in additional switching mechanisms as explained in the next paragraph. 
The three-dimensional setup which we are considering implies that the exchange interaction is not only sensitive to the magnitude of the applied fields, but also to their direction. We now give a brief overview of our results [23] for in-plane $\left(B_{\|}, E_{\|}\right)$and perpendicular $\left(B_{\perp}, E_{\perp}\right)$ fields; this setup is illustrated in Fig. 3a: (1) In-plane magnetic fields $B_{\|}$suppress $J$ exponentially; perpendicular fields in laterally coupled dots have the same effect. (2) Perpendicular magnetic fields $B_{\perp}$ reduce the exchange coupling between identically sized dots $\alpha_{0+}=\alpha_{0-}$ only slightly. However, if the two dots have different sizes, $a_{\mathrm{B}+}<a_{\mathrm{B}-}$, the behavior of $J\left(B_{\perp}\right)$ is no longer monotonic: Increasing $B_{\perp}$ from zero amplifies the exchange coupling $J$ until both electronic orbitals are magnetically compressed to approximately the same size, i.e. $B \approx 2 m \alpha_{0+} \omega_{z} c / e$. From this point on, $J$ decreases weakly, as for identically sized dots. (3) Perpendicular electric fields $E_{\perp}$ detune the single-dot levels, and thus reduce the exchange coupling; the same result was obtained for laterally coupled dots and in-plane electric fields [14]. (4) In-plane electric fields $E_{\|}$and different dot sizes provide another switching mechanism for $J$. The dots are shifted parallel to the field by $\Delta x_{ \pm}=E_{\|} / E_{0} \alpha_{0 \pm}^{2}$, where $E_{0}=\hbar \omega_{z} / e a_{\mathrm{B}}$. Thus, the larger dot is shifted a greater distance $\Delta x_{-}>\Delta x_{+}$and so the mean distance between the electrons grows as $d^{\prime}=\sqrt{d^{2}+A^{2}\left(E_{\|} / E_{0}\right)^{2}}>d$, taking $A=\left(\alpha_{0+}^{2}-\alpha_{0-}^{2}\right) / 2 \alpha_{0+}^{2} \alpha_{0-}^{2}$. Since the exchange coupling $J$ is exponentially sensitive to the inter-dot distance $d^{\prime}$, it is suppressed exponentially when an in-plane electric field is applied, $J \approx \exp \left[-2 A^{2}\left(E_{\|} / E_{0}\right)^{2}\right]$, which is illustrated in Fig. 3c. We have thus described an alternative to the switching mechanisms involving a magnetic field, namely an exponential switching mechanism for a quantum gate relying only on a tunable electric field.

\section{Single-spin rotations}

In addition to at least one (non-trivial) two-qubit gate (e.g. XOR or square-root-of-swap), it is required for quantum computation to perform one-qubit operations, which in the context of spin physics are equivalent to single-spin rotations. This amounts to exposing any specific qubit to a time-varying Zeeman coupling $\left(g \mu_{\mathrm{B}} S \cdot \boldsymbol{B}\right)(t)[14]$, which is controlled through the magnetic field $\boldsymbol{B}$ and/or the $\mathrm{g}$-factor $g$. Since only relative rotations are relevant, it is sufficient to rotate all spins of the system simultaneously (e.g. by an external field $B$ ). Relative rotations are then achieved by changing the Larmor frequency of individual spins.

Magnetic fields which vary strongly on the length scale given by the inter-dot distance can be generated using various techniques, e.g. with the magnetic tip of a scanning force microscope, a magnetic disk writing head, by placing the dots above a grid of current-carrying wires, or by placing a small wire coil above the dot. Alternatively one can use ESR techniques [14] to perform single-spin rotations, e.g. if a certain qubit has to be flipped (say from $|\uparrow\rangle$ to $|\downarrow\rangle$ ) one applies an ac magnetic field perpendicular to the $\uparrow$-axis which matches the Larmor frequency of that particular electron. 
The examples described in the following show that for both single-spin and two-spin operations, the control over the spin can be achieved through charge (i.e. orbital wave function) manipulation [4]. The coupling of the qubit-spins to a layer of material with a higher (or lower) g-factor can be achieved by changing the equilibrium position of the electron by electrical gating [6, 24] (cf. Fig. 1). If the electron wave function is pushed into a region with a different (effective) g-factor, a relative rotation around the direction of $\boldsymbol{B}$ by an angle of $\phi=\left(g^{\prime} B^{\prime}-g B\right) \mu_{\mathrm{B}} \tau / 2 \hbar$ is produced. In bulk semiconductors the free-electron value of the g-factor $g_{0}=2.0023$ is modified by spin-orbit coupling. Similarly, the g-factor can be drastically enhanced by doping the semiconductor with magnetic impurities [15, 16]. In confined structures such as quantum wells, wires, and dots, the g-factor is further modified and becomes sensitive to an external bias voltage [25]. Numerical analysis of a system with a layered structure (AlGaAs-GaAs-InAlGaAs-AlGaAs) has shown that by shifting an electron from one layer to another by electrical gating [26] the effective g-factor can be varied by about [24] $\Delta g_{\text {eff }} \approx 1$.

Localized magnetic fields appear to be more difficult to implement than the nearest-neighbor exchange coupling in some cases. In the following, we list a number of possibilities to use exclusively the exchange mechanism to achieve both the two-qubit and the single-qubit gates. By means of exchange coupling to ferromagnetic (FM) dots or to an FM layer (Fig. 1), the coupling of a single spin $\boldsymbol{S}$ to an effective Zeeman field $\left(g \mu_{\mathrm{B}} \boldsymbol{B}\right)(t)$ can be obtained. Regions (layers) with increased magnetic field can be provided by an FM material while an effective magnetic field can be produced e.g. with dynamically polarized nuclear spins (the Overhauser effect) [14]. Ferromagnetic dots can be made of magnetic metals (e.g. Co or Dy) or magnetic semiconductors, e.g. Mn-doped GaAs (in this case, exchange effects are equivalent to an effective g-factor description).

Finally, another possibility for exchange-only quantum computation consists of using an appropriate encoding for the qubits [27]. It turns out that the Heisenberg interaction Eq. (4) between the spins representing the qubits alone is sufficient to (exactly) perform any quantum computation if each qubit is encoded using (at least) three spins (instead of only one). A possible encoding for this is $\left|0_{\mathrm{L}}\right\rangle=|S\rangle|\uparrow\rangle,\left|1_{\mathrm{L}}\right\rangle=\sqrt{2 / 3}\left|T_{+}\right\rangle|\downarrow\rangle-\sqrt{1 / 3}\left|T_{0}\right\rangle|\uparrow\rangle$. One-qubit gates on the coded qubits can now be performed by applying the exchange interaction between the spins of the code; the Hamiltonian $H_{12}=J_{12} \boldsymbol{S}_{1} \cdot \boldsymbol{S}_{2}$ e.g. generates rotations about the $z$-axis for the coded state $\left|\psi_{\mathrm{L}}\right\rangle=\alpha\left|0_{\mathrm{L}}\right\rangle+\beta\left|1_{\mathrm{L}}\right\rangle$. Generally, it can be shown that any single-qubit rotation in code space can be performed with a sequence involving at most four steps of applying the exchange $H_{i j}$ between the adjacent spins $i$ and $j$ in the case where the spins are arranged in a line (i.e. with couplings $H_{12}$ and $H_{23}$ ), or three steps if the arrangement is in a "triangle" i.e. $H_{13}$ is also possible. A numerical analysis was performed in order to find the exact number of computational steps for performing XOR between two coded qubits [27] with the result that XOR involves 19 sequential applications of a nearest-neighbor ex- 
change interaction. In the case where parallel (simultaneous) exchange couplings are allowed, we find that 7 or 8 steps are required, depending on the geometry of the arrangement. These results quantify the expense in number of devices (a factor of three more) and computational steps (roughly a factor of ten more) if one wants to use the encoding method described above instead of implementing the control of local (real of effective) magnetic fields.

\section{Single-spin measurement}

At the end, the result of a quantum computation has to be read out, i.e. the state of each qubit (each individual electron spin) needs to be measured, e.g. in the $|\uparrow\rangle,|1\rangle$ basis. The direct detection of the tiny magnetic moment $\mu_{\mathrm{B}}$ of an individual electron spin is very difficult, but there exist several proposals for reading out single spins using more realistic methods. One of them is to use the tunneling of the electron into a supercooled paramagnetic dot $[4,6]$, thereby inducing a magnetization nucleation from the metastable phase into a ferromagnetic domain. The domain's magnetization direction is along the measured spin and can be detected by conventional methods and provides a $75 \%$ reliable result for the read out of the electron spin.

Another idea is to detect the spin of an electron via measuring charge, i.e. voltage or current [4]. It is known how to build electrometers with single-charge detection capabilities; resolutions down to $10^{-8}$ of one electron charge have been reported [28]. A straightforward concept yielding a potentially $100 \%$ reliable measurement requires a switchable "spin-filter" tunnel barrier which allows only, say, spin-up but no spin-down electrons to tunnel. When the measurement of a spin in a quantum dot is to be performed, tunneling between this dot and a second dot, connected to an electrometer, is switched on, but only spin-up electrons are allowed to pass (spin-filtering). Thus if the spin had been up, a charge would be detected in the second dot by the electrometer [4], and no charge otherwise. This is a positive operator valued measurement (POVM). Spin filtering and also spin-state measurements can be achieved by tunneling through a quantum dot [17].

\section{Production of spin-entangled electrons for quantum communication}

Two spins (qubits) are entangled if their wave function cannot be expressed as a tensor product of two single-spin wave functions. Many tasks in quantum communication require maximally entangled states of two qubits (EPR pairs) [29] such as the spin singlet $|S\rangle=(|\uparrow \downarrow\rangle-|\downarrow \uparrow\rangle) / \sqrt{2}$. The triplet $\left|T_{0}\right\rangle=(|\uparrow \downarrow\rangle+|\downarrow \uparrow\rangle) / \sqrt{2}$ is another maximally entangled state, while the other two triplets $\left|T_{ \pm}\right\rangle=|\uparrow \uparrow\rangle$, $|\downarrow \downarrow\rangle$ are not entangled. The quantum gate mechanism described in Secs. 4 and 5 are one possibility for producing such entangled states for electrons (we call in 
general such a device an entangler, for which several different realizations are conceivable). Here we present another method of producing entangled electrons making use of the Andreev tunneling at a superconductor-normal interface.

Since the Cooper pairs in conventional $s$-wave superconductors (SC) have singlet spin-wave functions [30] they can potentially act as a source of spin-entangled electrons. In a so-called Andreev process [31] two electrons of opposite spin tunnel coherently from an SC into a normal region while single-particle processes are suppressed. This is not enough for our purpose, however. In order to be useful for quantum communication, the two entangled electrons should be separated in space. It has been proposed to use two quantum dots in the Coulomb blockade [32] between the SC and each of the leads for achieving this goal (see Fig. 4). The tunneling amplitudes between $\mathrm{SC}$ and dots, and dots and leads, are denoted by $T_{\mathrm{SD}}$ and $T_{\mathrm{DL}}$. A bias voltage $\Delta \mu=\mu_{S}-\mu_{l}>0$ is applied in order to obtain transport of entangled electrons from the SC via the dots to the leads ( $\mu_{S}$ and $\mu_{l}=\mu_{1}=\mu_{2}$ are the chemical potentials of the $\mathrm{SC}$ and the leads). The chemical potentials $\epsilon_{1}$ and $\epsilon_{2}$ of the two quantum dots can be tuned by external gate voltages [32] such that the coherent tunneling of two electrons into different leads is at resonance. The resonance for the coherent tunneling of two electrons into the same lead is suppressed by the on-site Coulomb repulsion $U$ of a quantum dot.

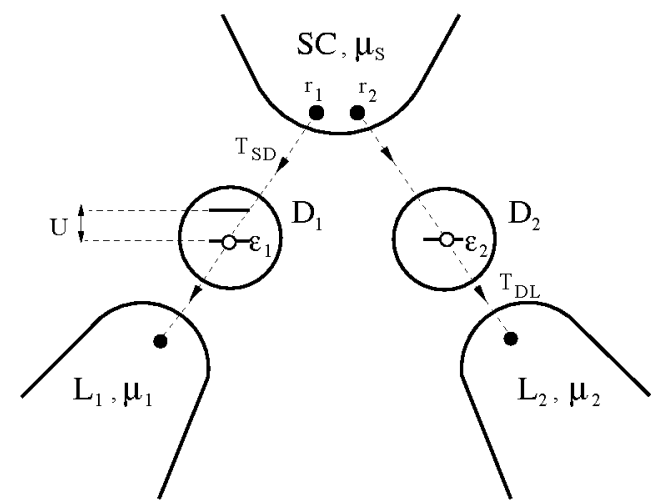

Fig. 4. Schematic drawing of the Andreev entangler. Two entangled electrons initially forming a Cooper pair hop with amplitude $T_{\mathrm{SD}}$ from two points $\boldsymbol{r}_{1}, \boldsymbol{r}_{2}$ of the superconductor, SC (distance $\delta r=\left|r_{1}-r_{2}\right|$ ), to two dots $D_{1,2}$ by means of the Andreev tunneling. The dots are coupled to normal leads $L_{1,2}$ with tunneling amplitude $T_{\mathrm{DL}}$. In order to maximize the efficiency of the device, we require asymmetric barriers, $\left|T_{\mathrm{SD}}\right| /\left|T_{\mathrm{DL}}\right| \ll 1$. The chemical potentials of the $\mathrm{SC}$ and leads are denoted $\mu_{l}$ and $\mu_{S}$.

We work at the resonances $\epsilon_{1}=\epsilon_{2}=\mu_{S}$ since then the total current and the desired suppression for tunneling into the same lead is maximized. In addition, in this way one can achieve the injection of the two electrons into separate leads at the same orbital energy - this turns out to be crucial for the detection of 
entanglement which is proposed in Sec. 10. It is most efficient to work in the regime where the dot levels $\epsilon_{n}$ have vanishing occupation probability. For this purpose we require that the quantum dot-lead coupling is much stronger than the SC-quantum dot coupling, i.e. $\left|T_{\mathrm{SD}}\right| \ll\left|T_{\mathrm{DL}}\right|$, so that electrons which enter the quantum dots from the SC will leave the quantum dots to the leads much faster than new electrons can be provided from the SC. The stationary occupation due to the coupling to the leads is indeed exponentially small if $\Delta \mu>k_{\mathrm{B}} T, T$ being the temperature and $k_{\mathrm{B}}$ the Boltzmann constant. In this asymmetric barrier case, the resonant dot levels $\epsilon_{n}$ can be occupied only during a virtual process.

In order to exclude spin-flip processes, we require that the number of electrons on each dot must be even, $N_{\mathrm{D}}=$ even, with total spin zero in the ground state. Even with $N_{\mathrm{D}}=$ even, there could be spin-flip processes which result in an excited state on the dot. However, those processes are strongly suppressed if $\delta \epsilon>k_{\mathrm{B}} T$ and $\delta \epsilon>\Delta \mu$, where $\delta \epsilon$ is the level spacing of the dot. Another mechanism for the loss of entanglement is given by electron-hole excitations in the leads during the resonant tunneling events. Again, this process is suppressed if the resonance width $\gamma_{l}=2 \pi \nu_{l}\left|T_{\mathrm{DL}}\right|^{2}$ is smaller than $\Delta \mu$ (for $\epsilon_{n} \simeq \mu_{S}$ ), where $\nu_{l}$ is the density of states per spin of the leads at the chemical potential $\mu_{l}$.

A further energy scale which enters the consideration is the superconducting energy gap $\Delta$. The time delay between subsequent coherent Andreev tunneling events of the two electrons of a Cooper pair is determined by $\Delta^{-1}$. In order to exclude single-electron tunneling where the creation of a quasiparticle in the $\mathrm{SC}$ is a final excited state we require that $\Delta \gg \Delta \mu$ and $\Delta \gg k_{\mathrm{B}} T$. Summarizing all above inequalities, we can specify the following regime of interest for entanglement production [7]:

$$
\Delta, U, \delta \epsilon>\Delta \mu>\gamma_{l}, k_{\mathrm{B}} T \quad \text { and } \quad \gamma_{l}>\gamma_{S} .
$$

In the regime specified by Eq. (10), we have calculated and compared the stationary charge current of two entangled electrons for two competing transport channels, first for the desired transport of the two entangled electrons each into different leads (current $I_{1}$ ) and second for the unwanted transport of both electrons into the same lead (current $I_{2}$ ). We have calculated the currents $I_{1}, I_{2}$ by making use of a T-matrix approach which is well adapted for describing Breit-Wigner resonances. The final result for the ratio of the two currents is [7]:

$$
\frac{I_{1}}{I_{2}}=\frac{2 \mathcal{E}^{2}}{\gamma^{2}}\left[\frac{\sin \left(k_{\mathrm{F}} \delta r\right)}{k_{\mathrm{F}} \delta r}\right]^{2} \mathrm{e}^{-2 \delta r / \pi \xi}, \quad \frac{1}{\mathcal{E}}=\frac{1}{\pi \Delta}+\frac{1}{U},
$$

where $\gamma=\gamma_{1}+\gamma_{2}$. The current $I_{1}$ becomes exponentially suppressed with increasing distance $\delta r=\left|r_{1}-r_{2}\right|$ between the tunneling points on the $\mathrm{SC}$, the scale given by the superconducting coherence length $\xi$. This does not pose severe restrictions for a conventional $s$-wave material with $\xi$ typically being on the order of $\mu \mathrm{m}$. In the important case $0 \leq \delta r \sim \xi$ the suppression is only polynomial $\propto 1 /\left(k_{\mathrm{F}} \delta r\right)^{2}$, with $k_{\mathrm{F}}$ being the Fermi wave vector in the SC. On the other hand, we see that the 
effect of the quantum dots consists in the suppression factor $(\gamma / \mathcal{E})^{2}$ for tunneling into the same lead. Thus, in addition to Eq. (10) we have to impose the condition $k_{\mathrm{F}} \delta r<\mathcal{E} / \gamma$, which can be satisfied for small dots with $\mathcal{E} / \gamma \approx 100$ and $k_{\mathrm{F}} \approx 1 \AA$. We would like to stress that the suppression (rather than only the absolute current) is maximized by working around the resonance $\epsilon_{n} \simeq \mu_{S}=0$. We remark that incoherent transport (sequential tunneling) is negligible as long as the scattering rate $\Gamma_{\varphi}$ is much smaller than $\gamma_{l}$ since $\left(I_{\text {seq }} / I_{\text {coh }}\right) \simeq\left(\Gamma_{\varphi} / \gamma_{l}\right)[33]$.

\section{Entangled electrons in a Fermi sea}

We now analyze the effect of interaction on entanglement [6, 8]. Entangled electrons which are transported in a conductor always interact with all other electrons in the conductor. Specifically, when we add an electron in state $q$ to a Fermi sea (lead), the quasiparticle weight of that state will be renormalized by $0 \leq z_{q} \leq 1$ (see below), i.e. some weight $1-z_{q}$ to find the electron in the original state $q$ will be distributed among all the other electrons $[6,8]$. This rearrangement of the Fermi system due to the Coulomb interaction happens very quickly, on a time scale given by the inverse plasmon frequency. We are now interested in quantifying this renormalization. More precisely, when a triplet/singlet electron pair ( $\mathrm{t}$ and $\mathrm{s}$ for short) is injected from an entangler into two leads 1 and 2, we obtain the state

$$
\left|\psi_{n n^{\prime}}^{\mathrm{t} / \mathrm{s}}\right\rangle=\frac{1}{\sqrt{2}}\left(a_{n \uparrow}^{\dagger} a_{n^{\prime} \downarrow}^{\dagger} \pm a_{n \downarrow}^{\dagger} a_{n^{\prime} \uparrow}^{\dagger}\right)\left|\psi_{0}\right\rangle
$$

with the filled Fermi sea $\left|\psi_{0}\right\rangle, n=(\boldsymbol{q}, l), \boldsymbol{q}$ the momentum of an electron, and $\boldsymbol{l}$ the lead number. The operator $a_{n \sigma}^{\dagger}$ creates an electron in state $n$ with spin $\sigma$. The propagation of the triplet or singlet, interacting with all other electrons in the Fermi sea, can be described by the 2-particle Green function $G^{\mathrm{t} / \mathrm{s}}(\mathbf{1 2}, \mathbf{3 4} ; t)=\left\langle\psi_{\mathbf{1 2}}^{\mathrm{t}}, t \mid \psi_{\mathbf{3 4}}^{\mathrm{t} / \mathrm{s}}\right\rangle$. If a triplet (singlet) is prepared at $t=0$, the probability for retrieving the same triplet (singlet) after time $t$ is found to be $P(t)=\left|G^{\mathrm{t} / \mathrm{s}}(\mathbf{1 2}, \mathbf{1 2} ; t)\right|^{2}$. Assuming sufficiently separated leads with negligible mutual interaction, we obtain $[6,8] P=z_{\mathrm{F}}^{4}$ for times much smaller than the quasiparticle lifetime (but larger than the inverse plasma frequency). For a spin-independent Hamiltonian with bare Coulomb interaction only and within random phase approximation (RPA) [34], the quasiparticle weight for a $2 \mathrm{DEG}$ is given by $[6,8] z_{\mathrm{F}}=1-r_{s}(1 / 2+1 / \pi)$, in leading order of the interaction parameter $r_{s}=1 / k_{\mathrm{F}} a_{\mathrm{B}}$, where $a_{\mathrm{B}}=\epsilon_{0} \hbar^{2} / m e^{2}$ is the Bohr radius and $k_{\mathrm{F}}$ the Fermi wave vector. In a typical GaAs 2DEG we have $a_{\mathrm{B}}=$ $10.3 \mathrm{~nm}$ and $r_{s}=0.614$, and thus we obtain $z_{\mathrm{F}}=0.665$, therefore the probability for recovering an injected singlet will only be around $P \approx 0.2$. However, for large electron density (leading to small $r_{s}$ ), $P$ will be closer to unity and some useful amount of entanglement can be preserved. This holds provided the spin-scattering effects are small, which is supported by experimental results where the electron spin has been transported phase-coherently over distances of up to $100 \mu \mathrm{m}$ [10]. 


\section{Noise of entangled electrons}

A stream of bosons exhibits "bunching" behavior in the correlations between particles ("noise") [35, 36]. Recently, the opposite behavior for fermions, "antibunching", was expected theoretically [37-39] and found experimentally [40], in particular for electrons. As pointed out recently [6] the noise of electrons in current-carrying wires is sensitive only to the symmetry of the orbital part of its wave function, at least if no spin-scattering processes are present. Thus, if we now consider a two-electron state, we expect antibunching for the triplet states, since they have an antisymmetric orbital wave function, whereas the orbital wave function associated with the spin singlet state is symmetric, and so we expect bunching. This leads to an observable decrease or increase in noise for electrons, depending on their common spin state, as we shall discuss next [8].

We assume that an entangler generates pairs of entangled electrons which are then injected into lead 1 and 2, one electron each, as shown in Fig. 5. A beam splitter is inserted in order to create two-particle interference effects in the sense that there is an equal probability amplitude for incoming electrons (from lead 1 or 2) to leave into lead 3 or 4 . The quantity of interest is then the noise, i.e. the current-current correlations, measured in leads 3 and/or 4.

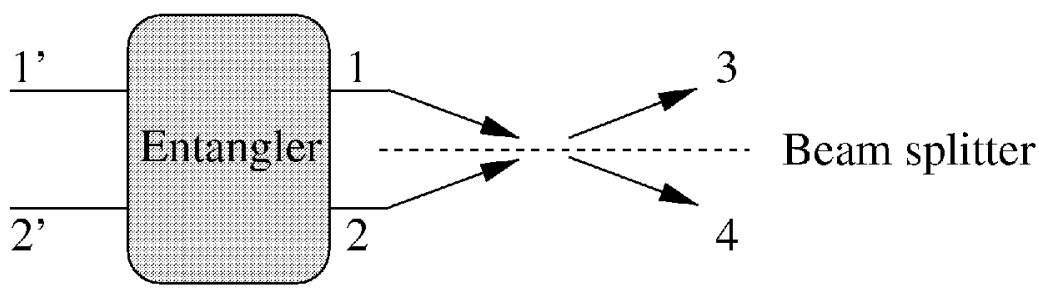

Fig. 5. Setup for measuring noise of entangled electrons. The entangler may take uncorrelated electrons from the Fermi leads $1^{\prime}$ and $2^{\prime}$ (this is not required for the Andreev entangler). Pairs of entangled electrons (singlet or triplet) are produced in the entangler and then injected into the leads 1 and 2, one electron per lead. The current of these two leads are then mixed with a beam splitter (to induce scattering interference) and the resulting noise is then measured in lead 3 and 4: no noise (antibunching) for triplets, whereas we obtain enhanced noise (bunching) for singlets.

The probability of recovering a singlet or triplet after injecting it into an interacting Fermi sea is reduced by a factor of $P=z_{\mathrm{F}}^{4}$, which depends on the carrier density. Except for this renormalization, the entanglement of the singlet or triplet is not affected by the interacting electrons in the filled Fermi sea. If $P=z_{\mathrm{F}}^{4}$ is close to one we can now calculate transport quantities using the standard scattering theory for non-interacting quasiparticles in a Fermi liquid [37]. We consider the entangled incident states $| \pm\rangle \equiv\left|\psi_{\mathbf{1 2}}^{\mathrm{t} / \mathrm{s}}\right\rangle$ with one electron per lead and the quantum numbers $n=\left(\varepsilon_{n}, n\right)$, where $\varepsilon_{n}$ is the electron energy. Considering a multiterminal 
conductor with density of states $\nu$, we assume that the leads consist of only one quantum channel; the generalization to more channels is straightforward.

For the spectral densities of the fluctuations between the leads $\alpha$ and $\beta$, we obtain $\delta I_{\alpha}=I_{\alpha}-\left\langle I_{\alpha}\right\rangle$ in the absence of backscattering and at zero frequency [8], $S_{\alpha \beta}(\omega)=\lim _{T \rightarrow \infty}(h \nu / T) \int_{0}^{T} \mathrm{~d} t \mathrm{e}^{\mathrm{i} \omega t} \operatorname{Re}\left\langle \pm\left|\delta I_{\alpha}(t) \delta I_{\beta}(0)\right| \pm\right\rangle$,

$$
S_{33}=S_{44}=-S_{34}=2 \frac{e^{2}}{h \nu} T(1-T)\left(1 \mp \delta_{\varepsilon_{1} \varepsilon_{2}}\right) .
$$

Here, the minus (plus) sign refers to the spin triplet (singlet) and $T$ is the transmission coefficient of the beam splitter. If two electrons with the same energies, $\varepsilon_{1}=\varepsilon_{2}$, in the singlet state are injected into the leads 1 and 2 , the shot noise is enhanced by a factor of two compared to the value for uncorrelated particles $[37,41], 2 e^{2} T(1-T) / h \nu$. This amplification of the noise arises from bunching of the electrons due to their symmetric orbital wave function, such that the electrons preferably appear in the same outgoing leads. If the electron pairs are injected as a triplet, an antibunching effect appears, completely suppressing the noise, i.e. $S(\omega=0)=0$. We stress that the sign of cross-correlations does not carry any signature of statistics, e.g. here the different signs of $S_{34}$ and $S_{33}=S_{44}$ [Eq. (13)] merely reflect current conservation and absence of backscattering. Since bunching appears only for a state with a symmetric orbital wave function, which cannot occur for unentangled states, measuring noise enhancement in the outgoing arms of the beamsplitter provides unique evidence for entanglement [8]. A crucial requirement is $\varepsilon_{1}=\varepsilon_{2}$, which can be satisfied for finite-size systems of length $L$ (including the beam splitter), with discrete spectrum $\varepsilon$ such that $\Delta \varepsilon>k_{\mathrm{B}} T>\gamma$ or $L<\hbar v_{\mathrm{F}} / k_{\mathrm{B}} T \approx 2 \mu \mathrm{m}$ at $1 \mathrm{~K}$. Here, $\gamma$ is the level width of $\varepsilon$ caused by the coupling to the current reservoirs. Furthermore, inelastic scattering should be negligible since it can lead to a violation of the condition $\varepsilon_{1}=\varepsilon_{2}$ by changing the energy of one of the particles. Therefore one has to work at low temperatures where $\ell_{\text {inelastic }}>L$.

\section{References}

[1] M.A. Nielsen, I.L. Chuang, Quantum Computation and Quantum Information, Cambridge University Press, Cambridge 2000.

[2] D.P. DiVincenzo, in Ref. [3], p. 771.

[3] Experimental Proposals for Quantum Computation, Special Focus Issue of Fortschritte der Physik, Vol. 48, Eds. S. Braunstein, H.-K. Lo, Wiley, Berlin 2000.

[4] D. Loss, D.P. DiVincenzo, Phys. Rev. A 57, 120 (1998); http://www.arxiv.org/abs/cond-mat/9701055.

[5] D.P. DiVincenzo, Phys. Rev. A 51, 1015 (1995).

[6] D.P. DiVincenzo, D. Loss, J. Magn. Magn. Mater. 200, 202 (1999); http://www.arxiv.org/abs/cond-mat/9901137.

[7] P. Recher, E.V. Sukhorukov, D. Loss, Phys. Rev. B 63, 165314 (2001). 
[8] G. Burkard, D. Loss, E.V. Sukhorukov, Phys. Rev. B 61, R16303 (2000).

[9] D. Bouwmeester, J.-W. Pan, K. Mattle, M. Eibl, H. Weinfurter, A. Zeilinger, Nature 390, 575 (1997); D. Boschi, S. Branca, F. De Martini, L. Hardy, S. Popescu, Phys. Rev. Lett. 80, 1121 (1998).

[10] J.M. Kikkawa, I.P. Smorchkova, N. Samarth, D.D. Awschalom, Science 277, 1284 (1997); J.M. Kikkawa, D.D. Awschalom, Phys. Rev. Lett. 80, 4313 (1998); D.D. Awschalom, J.M. Kikkawa, Phys. Today 52, 33 (1999).

[11] J.A. Gupta, D.D. Awschalom, X. Peng, A.P. Alivisatos, Phys. Rev. B 59, R10421 (1999).

[12] H.-A. Engel, D. Loss, Phys. Rev. Lett. 86, 4648 (2001).

[13] A.V. Khaetskii, Y.V. Nazarov, Phys. Rev. B 61, 12639 (2000); http://www.arxiv.org/abs/cond-mat/0003513; A.V. Khaetskii, Physica E 10, 27 (2001).

[14] G. Burkard, D. Loss, D.P. DiVincenzo, Phys. Rev. B 59, 2070 (1999).

[15] R. Fiederling, M. Keim, G. Reuscher, W. Ossau, G. Schmidt, A. Waag, L.W. Molenkamp, Nature 402, 787 (1999).

[16] Y. Ohno, D.K. Young, B. Beschoten, F. Matsukura, H. Ohno, D.D. Awschalom, Nature 402, 790 (1999).

[17] P. Recher, E.V. Sukhorukov, D. Loss, Phys. Rev. Lett. 85, 1962 (2000).

[18] A. Barenco, C.H. Bennett, R. Cleve, D.P. DiVincenzo, N. Margolus, P. Shor, T. Sleator, J.A. Smolin, H. Weinfurter, Phys. Rev. A 52, 3457 (1995).

[19] S. Tarucha, D.G. Austing, T. Honda, R.J. van der Hage, L.P. Kouwenhoven, Phys. Rev. Lett. 77, 3613 (1996).

[20] X. Hu, S. Das Sarma, Phys. Rev. A 61, 062301 (2000).

[21] R.J. Luyken, A. Lorke, M. Haslinger, B.T. Miller, M. Fricke, J.P. Kotthaus, G. Medeiros-Ribiero, P.M. Petroff, Physica E 2, 704 (1998).

[22] D.G. Austing, T. Honda, K. Muraki, Y. Tokura, S. Tarucha, Physica B 249-251, 206 (1998).

[23] G. Burkard, G. Seelig, D. Loss, Phys. Rev. B 62, 2581 (2000).

[24] D.P. DiVincenzo, G. Burkard, D. Loss, E. Sukhorukov, in: Quantum Mesoscopic Phenomena and Mesoscopic Devices in Microelectronics, Eds. I.O. Kulik, R. Ellialtoglu, NATO ASI, Kluwer Academic Publ. Dordecht 2000, p. 399; see http://www.arxiv.org/abs/cond-mat/99112445.

[25] E.L. Ivchenko, A.A. Kiselev, M. Willander, Solid State Commun. 102, 375 (1997).

[26] K. Ensslin, private communication.

[27] D.P. DiVincenzo, D. Bacon, J. Kempe, G. Burkard, K.B. Whaley, Nature 408, 339 (2000).

[28] M. Devoret, D. Estève, Ch. Urbina, Nature (London) 360, 547 (1992).

[29] A.K. Ekert, Phys. Rev. Lett. 67, 661 (1991).

[30] J.R. Schrieffer, Theory of Superconductivity, Benjamin/Cummings, New York 1964.

[31] F.W.J. Hekking, L.I. Glazman, K.A. Matveev, R.I. Shekhter, Phys. Rev. Lett. 70, 4138 (1993). 
[32] L.P. Kouwenhoven, G. Schön, L.L. Sohn, Mesoscopic Electron Transport, NATO ASI Series E, Vol. 345, Kluwer Academic Publ., Dordrecht 1997.

[33] S. Datta, Electronic Transport In Mesoscopic Systems, Cambridge University Press, Cambridge 1995, p. 260.

[34] G.D. Mahan, Many Particle Physics, 2nd ed., Plenum, New York 1993.

[35] R. Loudon, Phys. Rev. A 58, 4904 (1998).

[36] R. Hanbury Brown, R.Q. Twiss, Nature (London) 177, 27 (1956).

[37] M. Büttiker, Phys. Rev. Lett. 65, 2901 (1990); Phys. Rev. B 46, 12485 (1992).

[38] T. Martin, R. Landauer, Phys. Rev. B 45, 1742 (1992).

[39] E.V. Sukhorukov, D. Loss, Phys. Rev. B 59, 13054 (1999).

[40] R.C. Liu, B. Odom, Y. Yamamoto, S. Tarucha, Nature 391, 263 (1998); M. Henny, S. Oberholzer, C. Strunk, T. Heinzel, K. Ensslin, M. Holland, C. Schönenberger, Science 284, 296 (1999); W.D. Oliver, J. Kim, R.C. Liu, Y. Yamamoto, Science 284, 299 (1999).

[41] V.A. Khlus, Zh. Eksp. Teor. Fiz. 93, 2179 (1987). 